\title{
Structure of a New Antioxidative Phenolic Acid from Oregano (Origanum vulgare L.)
}

\author{
Hiroe KikUZaki and Nobuji NaKaTani* \\ Department of Food and Nutrition, Faculty of Science of Living, \\ Osaka City University, Sumiyoshi-ku, Osaka 558, Japan \\ Received September 19, 1988
}

\begin{abstract}
Five antioxidative phenolic acids were isolated from the leaves of oregano (Origanum vulgare L.). The structure of a new compound (5a) was determined to be 2-caffeoyloxy-3-[2-(4-hydroxybenzy)4,5-dihydroxy]phenylpropionic acid on the basis of spectroscopic evidence. Especially, phenyl glucoside (1) and 5a showed an activity comparable to that of BHA.
\end{abstract}

Much attention has been paid to spices as a resource of natural antioxidants for depressing the rancidity of fats and oils in food. Oregano (Origanum vulgare L.), one of the herbal spices belonging to the Family Labiatae, is known to have antioxidative activity. Chipault et al. examined the antioxidative effect of petroleum ether and ethyl alcohol soluble fractions extracted from many spices, and found that oregano had antioxidative properties in both fractions. ${ }^{1)}$ They also reported in another article that oregano was not particularly effective in lard, but was most effective in mayonnaise and French dressing in all the tested samples. ${ }^{2,3)}$ As a part of a study on natural antioxidants, we have previously reported a new antioxidative glucoside (1) from this plant. ${ }^{4}$ In this paper, we describe the isolation and structural elucidation of an additional new phenolic acid from oregano.

Dried leaves of oregano were successively extracted with dichloromethane and methanol. The ethyl acetate soluble part of the methanol extract showed strong antioxidative activity against linoleic acid in the ferric thiocyanate method. ${ }^{5)}$ This ethyl acetate soluble fraction was dissolved in $20 \%$ methanol in water and subjected to column chromatography on polyamide. Compound 1, protocatechuic acid (2) and caffeic acid (3) were isolated from the fractions eluted with $20 \%, 50 \%$ and $80 \%$ methanol in water, respectively. The $0.5 \%$ formic acid in methanol fraction was further purified by silica gel and Sephadex LH-20 column<smiles>O=C(Cc1ccc(OC2OCC3C(CC(O)C(O)C(O)C3O)O2)cc1)c1ccc(O)c(O)c1</smiles><smiles>O=C(O)c1ccc(O)c(O)c1</smiles>
?<smiles>O=C(O)C=Cc1ccc(O)c(O)c1</smiles>
3

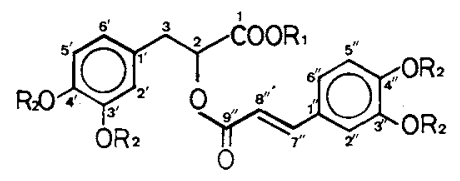

这: $R_{1}=R_{2}=H$ 4D: $\mathrm{R}_{1}=\mathrm{H}_{2} \mathrm{R}_{2}=\mathrm{COCH}_{3}$ 4C: $\mathrm{R}_{1}=\mathrm{CH}_{3}, \mathrm{R}_{2}=\mathrm{COCH}_{3}$

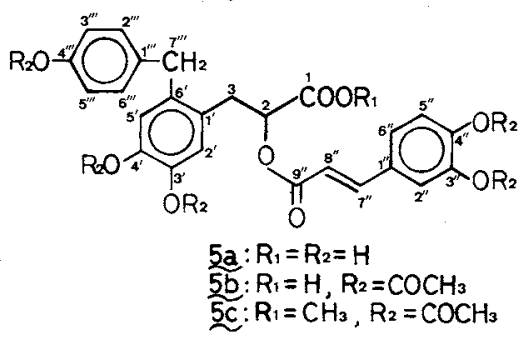

* Address correspondence to this author. 
chromatography and by repeated preparative HPLC to give compounds $\mathbf{4 a}$ and $\mathbf{5 a}$. Compound 4a was identified as rosmarinic acid on the basis of the spectral and physical data of its acetyl derivative (4b) and of its methyl ester (4c) which was synthesized from $4 b^{6 \sim 8)}$

Compound 5a was an off-white amorphous powder, mp $152 \sim 155^{\circ} \mathrm{C}$. SI-MS of 5 a gave a molecular size of 466 . The IR spectrum of this compound showed the presence of hydroxyl groups at $3500 \mathrm{~cm}^{-1}$ and in the region of $3100 \sim 2400 \mathrm{~cm}^{-1}$. The latter absorption and a band at $1680 \mathrm{~cm}^{-1}$ suggested the presence of a conjugated carboxylic function. The ${ }^{13} \mathrm{C}$ NMR spectrum of $\mathbf{5 a}$ revealed that this compound bore 25 carbons, which consisted of two methylene, one methine, two olefinic, eighteen aromatic and two carbonyl carbons. In the ${ }^{1} \mathrm{H}$ NMR spectrum, two double doublets at $\delta 2.97$ $(J=3.4,14.0 \mathrm{~Hz})$ and $3.11(J=9.7,14.0 \mathrm{~Hz})$ were attributed to the geminal protons of the benzylic methylene, which were coupled with a double doublet at $\delta 5.09$ assignable to the oxymethine proton. Two doublets at $\delta 6.23$ and $7.52(J=15.9 \mathrm{~Hz})$ and three aromatic protons $[\delta 6.77(\mathrm{~d}, J=8.3 \mathrm{~Hz}), 6.93(\mathrm{dd}, J=2.0$, $8.3 \mathrm{~Hz}), 7.03(\mathrm{~d}, J=2.0 \mathrm{~Hz})]$ suggested the presence of a trans-caffeoyl moiety, which was also supported by $\lambda_{\max }$ at $290,328 \mathrm{~nm}$ in the UV spectrum and by ${ }^{13} \mathrm{C}-\mathrm{NMR}$ data (Table II). These data indicated that compound $\mathbf{5 a}$ had a similar structure to rosmarinic acid (4a). In comparison with the ${ }^{1} \mathrm{H}-\mathrm{NMR}$ spectrum of 4a, two singlets at $\delta 6.51$ and 6.75 were observed in the spectrum of $\mathbf{5 a}$, instead of the characteristic ABX-type aromatic proton signals at $\delta 6.62(\mathrm{dd}, J=2.0,8.1 \mathrm{~Hz}), 6.68(\mathrm{~d}$, $J=8.1 \mathrm{~Hz})$ and $6.76(\mathrm{~d}, J=2.0 \mathrm{~Hz})$ in that of 4a. It is deduced from this finding that a 1,2,4,5-tetrasubstituted aromatic ring was present in the molecule of $\mathbf{5 a}$ in place of the 1,3,4trisubstituted one of $\mathbf{4 a}$. In addition, a pair of two ortho-coupling protons were observed at $\delta 6.67$ and 6.95 , accompanied with diphenyl methylene protons at $\delta 3.89$. Therefore, it was suggested that a 4-hydroxybenzylic moiety was attached to C-6' of rosmarinic acid (4a). This fact was further supported by the presence of signals at $\delta 38.2,116.2(2 \mathrm{C}), 130.7(2 \mathrm{C}), 133.6$ and 156.4 in the ${ }^{13} \mathrm{C}-\mathrm{NMR}$ spectrum, and by the molecular size of $466\left(\mathrm{M}^{+}\right)$, which was 106 mass $\left(\mathrm{C}_{7} \mathrm{H}_{6} \mathrm{O}\right)$ larger than that of rosmarinic acid. The assignments of $\mathrm{H}-2^{\prime}$ and $\mathrm{H}-5^{\prime}$ were confirmed by the irradiation technique in the ${ }^{1} \mathrm{H}-\mathrm{NMR}$ measurement. When the methylene protons at $\delta 2.97$ and $3.11(\mathrm{H}-3)$ were irradiated, the signal at $\delta 6.75$ was sharpened. On the other hand, when the methylene protons at $\delta 3.89\left(\mathrm{H}-7^{\prime \prime}\right)$ were irradiated, the two-proton doublet at $\delta 6.95\left(\mathrm{H}-2^{\prime \prime \prime}, 6^{\prime \prime \prime}\right)$ and the singlet at $\delta 6.51$ were sharpened. This confirmed that the singlet at $\delta 6.51$ was attributed to $\mathrm{H}^{-5^{\prime}}$, and the other singlet at $\delta 6.75$ to $\mathrm{H}-2^{\prime}$. The upfield shift $(0.24 \mathrm{ppm})$ of $\mathrm{H}-5^{\prime}$ compared with $\mathrm{H}-2^{\prime}$ is considered to have been due to the shielding effect of the 4-hydroxybenzyl group at C-6'.

The ${ }^{13} \mathrm{C}-\mathrm{NMR}$ spectral data of $\mathbf{4 a}$ and $\mathbf{5 a}$ are listed in Table II, the assignment of signals being determined by the ${ }^{13} \mathrm{C}-{ }^{1} \mathrm{H}$ COSY technique. The chemical shifts of $\mathrm{C}-1^{\prime} \sim 6^{\prime}$ of the benzene ring revealed the substitution of the 4-

Table I. ${ }^{1}$ H-NMR DATA OF COMPOUNDS 4a AND 5a $\left(400.5 \mathrm{MHz}\right.$, methanol- $\left.d_{4}\right)$

\begin{tabular}{cll}
\hline Proton no. & $\mathbf{4 a}$ & $\mathbf{5 a}$ \\
\hline $\mathrm{H}-2$ & $5.12 \mathrm{dd}$ & $5.09 \mathrm{dd}$ \\
$\mathrm{H}-3 \mathrm{a}$ & $2.97 \mathrm{dd}$ & $2.97 \mathrm{dd}$ \\
$\mathrm{H}-3 \mathrm{~b}$ & $3.11 \mathrm{dd}$ & $3.11 \mathrm{dd}$ \\
$\mathrm{H}-2^{\prime}$ & $6.76 \mathrm{~d}$ & $6.75 \mathrm{~s}$ \\
$\mathrm{H}-5^{\prime}$ & $6.68 \mathrm{~d}$ & $6.51 \mathrm{~s}$ \\
$\mathrm{H}-6^{\prime}$ & $6.62 \mathrm{dd}$ & \\
$\mathrm{H}-2^{\prime \prime}$ & $7.03 \mathrm{~d}$ & $7.03 \mathrm{~d}$ \\
$\mathrm{H}-5^{\prime \prime}$ & $6.77 \mathrm{~d}$ & $6.77 \mathrm{~d}$ \\
$\mathrm{H}-6^{\prime \prime}$ & $6.92 \mathrm{dd}$ & $6.93 \mathrm{dd}$ \\
$\mathrm{H}-7^{\prime \prime}$ & $7.52 \mathrm{~d}$ & $7.52 \mathrm{~d}$ \\
$\mathrm{H}-8^{\prime \prime}$ & $6.26 \mathrm{~d}$ & $6.23 \mathrm{~d}$ \\
$\mathrm{H}-2^{\prime \prime \prime}$ & & $6.95 \mathrm{~d}$ \\
$\mathrm{H}-3^{\prime \prime \prime}$ & & $6.67 \mathrm{~d}$ \\
$\mathrm{H}-5^{\prime \prime \prime}$ & & $6.67 \mathrm{~d}$ \\
$\mathrm{H}-6^{\prime \prime \prime}$ & & $6.95 \mathrm{~d}$ \\
$\mathrm{H}-7^{\prime \prime \prime}$ & & $3.89 \mathrm{~s}$ \\
\hline
\end{tabular}

$J(\mathrm{~Hz}) \quad 4 \mathrm{a}: \quad 2,3 \mathrm{a}=9.2 ; 2,3 \mathrm{~b}=3.5 ; 3 \mathrm{a}, 3 \mathrm{~b}=14.3 ; 2$, $6^{\prime}=2.0 ; \quad 5^{\prime}, \quad 6^{\prime}=8.1 ; \quad 2^{\prime \prime}, \quad 6^{\prime \prime}=2.0 ; \quad 5^{\prime \prime}$, $6^{\prime \prime}=8.2 ; 7^{\prime \prime}, 8^{\prime \prime}=15.9$

5a: $2,3 \mathrm{a}=9.7 ; 2,3 \mathrm{~b}=3.4 ; 3 \mathrm{a}, 3 \mathrm{~b}=14.0 ; 2^{\prime \prime}$, $6^{\prime \prime}=2.0 ; 5^{\prime \prime}, 6^{\prime \prime}=8.3 ; 7^{\prime \prime}, 8^{\prime \prime}=15.9 ;\left(2^{\prime \prime \prime}\right.$, $\left.6^{\prime \prime \prime}\right),\left(3^{\prime \prime \prime}, 5^{\prime \prime \prime}\right)=8.5$ 
hydroxybenzyl group at $\mathrm{C}-6^{\prime}$ and two hydroxyl groups at C-3' and C-4'. This was confirmed by the chemical shifts of the meth-

Table II. ${ }^{13} \mathrm{C}$-NMR ChEMICAL Shifts of Compoundos 4a AND 5a $\left(100.7 \mathrm{MHz}\right.$, methanol- $\left.d_{4}\right)$

\begin{tabular}{|c|c|c|}
\hline Carbon no. & $4 a$ & $5 a$ \\
\hline$C-1$ & 176.9 & 176.9 \\
\hline C-2 & 76.7 & 75.8 \\
\hline C-3 & 38.4 & 35.1 \\
\hline$C-1^{\prime}$ & 130.6 & $127.8^{a}$ \\
\hline$C-2^{\prime}$ & 117.6 & 118.6 \\
\hline C-3' & 146.0 & $144.9^{b}$ \\
\hline$C-4^{\prime}$ & 145.0 & $144.4^{b}$ \\
\hline$C-5^{\prime}$ & 116.3 & 118.5 \\
\hline C- $6^{\prime}$ & 121.8 & $132.8^{c}$ \\
\hline C-1" & 127.9 & $127.9^{a}$ \\
\hline $\mathrm{C}-2^{\prime \prime}$ & 115.2 & 115.3 \\
\hline C-3" & 146.7 & 146.8 \\
\hline C-4" & 149.5 & 149.6 \\
\hline C-5" & 116.5 & 116.5 \\
\hline C-6" & 123.0 & 123.0 \\
\hline$C-7^{\prime \prime}$ & 147.1 & 147.3 \\
\hline C-8" & 115.2 & 115.0 \\
\hline $\mathrm{C}-9^{\prime \prime}$ & 168.9 & 168.7 \\
\hline C-1"' & & $133.6^{c}$ \\
\hline C-2"' & & 130.7 \\
\hline C-3"' & & 116.2 \\
\hline C-4"' & & 156.4 \\
\hline$C-5^{\prime \prime \prime}$ & & 116.2 \\
\hline C- $6^{\prime \prime \prime}$ & & 130.7 \\
\hline$C-7^{\prime \prime \prime}$ & & 38.2 \\
\hline
\end{tabular}

a.b.c Assignments are interchangeable. ylene carbons of C-3 and C-7"'. A comparison of 5a with $4 \mathbf{a}$ showed an upfield shift (3.3 ppm) of C-3 due to the steric compression effect from the adjacent alkyl group. ${ }^{9}$ On the contrary, if a hydroxyl group was located at an ortho position to an alkyl substituent on the aromatic ring, an upfield shift of more than 5 ppm of the benzylic carbon would have been observed. ${ }^{10 \prime}$ In respect of C- $7^{\prime \prime \prime}$, the chemical shift value $(\delta 38.2)$ was close to that of diphenylmethane. ${ }^{11)}$ Thus, it must be concluded that C-6' was substituted by the 4-hydroxybenzyl group.

Compound 5a showed a specific rotation of $+76.7^{\circ}$ (methanol), which was the same behavior as that of rosmarinic acid $\left([\alpha]_{D}^{25}\right.$ : $\left.+70.8^{\circ}\right)$. Therefore, the stereochemistry at C-2 is proposed to have been of $R$ configuration. ${ }^{12)}$

Acetylation of 5a with acetic anhydride and pyridine gave a pentaacetate $(\mathbf{5 b})$. The IR spectrum newly showed an absorption band of acetates at $1760 \mathrm{~cm}^{-1}$, with disappearance of the peak at $3500 \mathrm{~cm}^{-1}$ in that of 5a. The signals of five phenolic acetates were observed at $\delta 2.22,2.24(\mathrm{OAc} \times 2), 2.29$ and 2.30 in the ${ }^{1} \mathrm{H}-\mathrm{NMR}$ spectrum, and five carbonyl carbons of acetates were obtained at $168.5,168.6(2 \mathrm{C})$, 168.7 and 169.6 in the ${ }^{13} \mathrm{C}-\mathrm{NMR}$ spectrum. The ${ }^{1} \mathrm{H}-\mathrm{NMR}$ and ${ }^{13} \mathrm{C}-\mathrm{NMR}$ data supported the structure of $\mathbf{5 b}$.

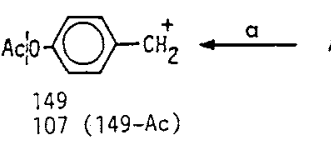<smiles>[CH2+]c1ccc(Cl)c(O)c1</smiles>

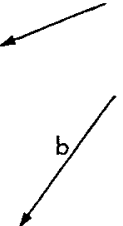

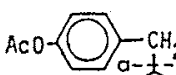

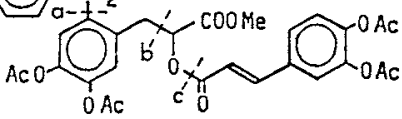

$548\left(M^{+}-A C\right)$ $605(648-A C)$

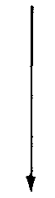

Mclafferty rearrangement<smiles>[CH2+]c1cc(Cc2ccc(OC(C)(C)C)cc2)c([CH2+])c(OC(C)=O)c1</smiles>

$271(323-\mathrm{Ac})$ $229(271-\mathrm{Ac})$

180
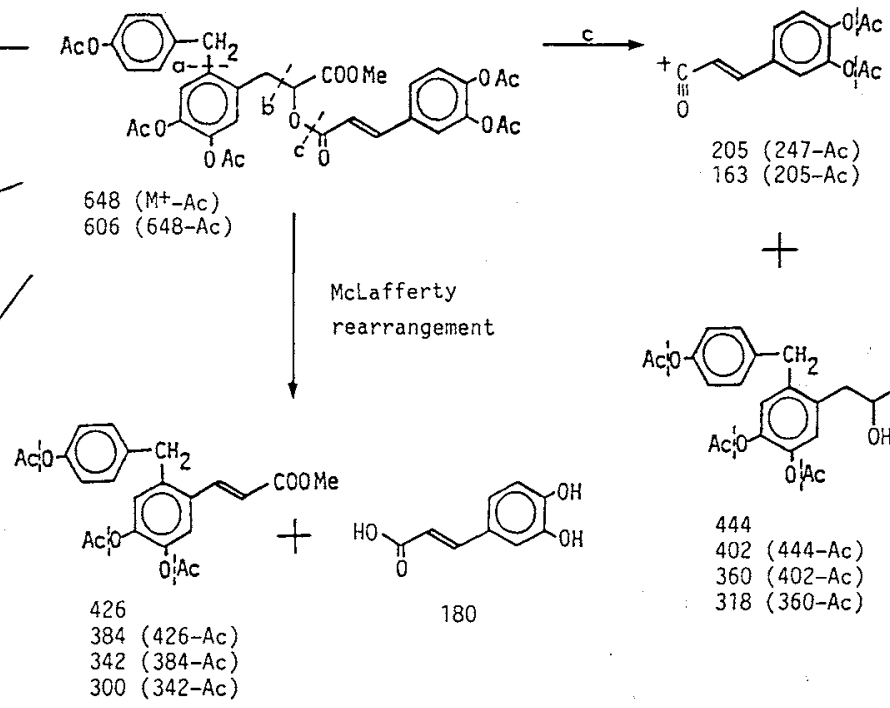

$205(247-A c)$
$163(205-A c)$
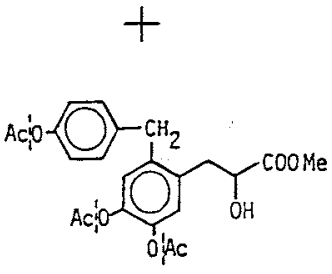

444

$402(444-A c)$

$360(402-A C)$

$318(360-A c)$

Fig. 1. Mass Fragmentation Pattern of Compound 5e. 


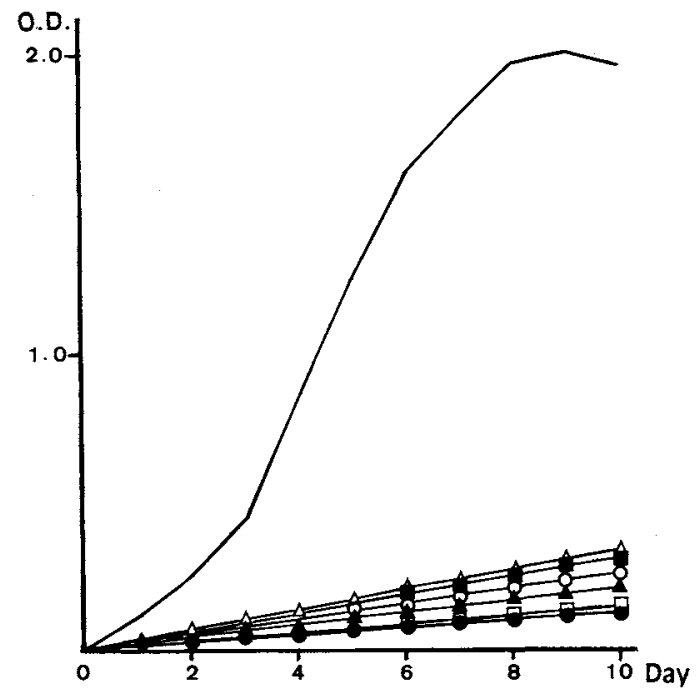

Fig. 2. Antioxidative Activity of Compounds $1 \sim 5 \mathbf{5 a}$ Measured by the Ferric Thiocyanate Method (conc. $0.02 \%$ ). $\square, \mathbf{1} ; \bigcirc, 2,4 \mathbf{4 a} ; \mathbf{\boldsymbol { C }}, \mathbf{3} ; \mathbf{\Delta}, \mathbf{5 a} ; \boldsymbol{\ominus}, \mathrm{BHA} ; \triangle, \alpha$-tocopherol;
- control.

Methylation of compound $\mathbf{5 b}$ with ethereal diazomethane afforded compound $\mathbf{5 c}$. The signal of a methyl group newly appeared at $\delta 3.74$ in the ${ }^{1} \mathrm{H}$-NMR spectrum and at $\delta 52.5$ in the ${ }^{13} \mathrm{C}-\mathrm{NMR}$ spectrum. The IR spectrum of $\mathbf{5 c}$ revealed the loss of a hydroxyl group in the region of $2400 \sim 3100 \mathrm{~cm}^{-1}$. The mass fragmentation pattern of $\mathbf{5 c}$ is shown in Fig. 1. The cleavage at bond a gave the 4-acetoxybenzyl cation $(m / z 149)$ and its deacetyl ion $(m / z 107)$, and the cleavage at bond $\mathbf{b}$ produced the benzyl cation $(m / z 229)$ as a main ion peak. The other major fragmentation pathway (c) yielded the acylonium ion $(\mathrm{m} / z 205$ and 163$)$, and $m / z$ 440, 402, 360 and 318. A McLafferty rearrangement gave the fragments of $m / z 426$ and 180 , the former being degradated stepwise by deacetylation to a stable ion of $m / z 300$.

Based on all the above data, the structure of 5a was established to be 2-caffeoyloxy-3-[2-(4hydroxybenzyl)-4,5-dihydroxylphenylpropionic acid.

The antioxidative activity of the five phenolic compounds isolated from oregano was measured by the ferric thiocyanate method (Fig. 2). All of them were more active than $\alpha$ - tocopherol, a common natural antioxidant. Among them, two new compounds (1 and 5a) had an activity comparable with that of BHA, a synthetic antioxidant. It is noteworthy that $\mathbf{1}$ showed stronger activity than protocatechuic acid (2), which was composed of the acyl moiety of 1, and that 5a seemed to be more effective than rosmarinic acid (4a), which occupied the most part of the structure of $\mathbf{5 a}$.

\section{Experimental}

Melting points ( $\mathrm{mp}$ ) were measured with a Yanagimoto micro-melting point apparatus and were uncorrected. UV absorption spectra were determined on a Hitachi 220 spectrophotometer, and IR spectra were recorded by a Jasco IR-S. ${ }^{1} \mathrm{H}-\mathrm{NMR} \quad(400.5 \mathrm{MHz})$ and ${ }^{13} \mathrm{C}-\mathrm{NMR}$ $(100.7 \mathrm{MHz})$ spectra were determined on a JEOL GX-400 using TMS as an internal standard. MS were obtained on a Hitachi M-2000, and optical rotation values were measured with a Union PM-101. Column chromatography was performed using Wako polyamide C-200, Merck silica gel $60(70 \sim 230$ mesh) and Pharmacia Sephadex LH-20, and TLC was carried out using silica gel GF-254. HPLC was carried out with a Shimadzu LC-4A for analysis and a Shimadzu LC-6A for preparation

Extraction and isolation. The $\mathrm{MeOH}$ extract $(88.0 \mathrm{~g})$ of dried leaves of oregano ( $1 \mathrm{~kg}$ ) was separated into $\mathrm{CH}_{2} \mathrm{Cl}_{2}$, AcOEt and $\mathrm{H}_{2} \mathrm{O}$ soluble fractions. The AcOEt soluble part $(7.5 \mathrm{~g}$ ) was dissolved in $20 \% \mathrm{MeOH}$ aq. and subjected to polyamide column chromatography, which was successively eluted with $20 \%, 50 \%$ and $80 \% \mathrm{MeOH}$ aq. and MeOH. Compounds 1 ( $2.33 \mathrm{~g}), 2$ (132 mg) and 3 (106 mg) were isolated from the $20 \%, 50 \%$ and $80 \% \mathrm{MeOH}$ aq. fractions, respectively. Finally, the column was eluted with a mixture of $\mathrm{MeOH}-\mathrm{HCOOH}(995: 5)$. This fraction was rechromatographed on silica gel using AcOEt-MeOH$\mathrm{H}_{2} \mathrm{O}(21: 4: 3 \mathrm{v} / \mathrm{v})$ as an eluent to separate into four fractions.

'Compound 4a. The third fraction $(1.14 \mathrm{~g})$ was rechromatographed on a Sephadex LH-20 column using $80 \%$ EtOH aq. as an eluent to afford an off-white amorphous powder $(790 \mathrm{mg})$. This compound was identified as rosmarinic acid, $\left.{ }^{6} \sim 8\right)$ mp $194 \sim 195^{\circ} \mathrm{C}$. UV $\hat{i}_{\max }(\mathrm{MeOH}) \mathrm{nm}$ $(\log c): 291(4.01), 327(4.08) \cdot[\alpha]_{D}^{25}:+70.8^{\circ} \quad(c=0.24$, $\mathrm{MeOH})$. IR $v_{\max }(\mathrm{Nujol}) \mathrm{cm}^{-1}: 3600,3400 \sim 2400,1685$, 1600, 1515. SI-MS: $453(\mathrm{M}+\mathrm{H}+\mathrm{G})^{+}, 361(\mathrm{M}+\mathrm{H})^{+} 198$.

Tetraacetyl rosmarinic acid. To a solution of $30 \mathrm{mg}$ of compound $4 \mathrm{a}$ in pyridine $(1 \mathrm{ml})$, acetic anhydride $(1 \mathrm{ml})$ was added, and the mixture was allowed to stand overnight at room temperature. The reaction mixture was poured into cold $2 \mathrm{~N}-\mathrm{HCl}$ and then extracted with AcOEt. 
The organic layer was washed with $\mathrm{H}_{2} \mathrm{O}$, dried over anbydrous $\mathrm{MgSO}_{4}$ and evaporated to dryness. Recrystallization of this residue from benzene gave colorless needles of compound $4 \mathrm{~b}(28 \mathrm{mg}), \mathrm{mp} 119 \sim 120^{\circ} \mathrm{C}$. UV $\hat{i}_{\text {max }}(\mathrm{MeOH}) \mathrm{nm}(\log \varepsilon): 279(4.41) \cdot[\alpha]_{\mathrm{D}}^{25}:+46.2^{\circ}(c=0.26$, $\mathrm{MeOH}) . I R v_{\max }(\mathrm{Nujol}) \mathrm{cm}^{-1}: 3400 \sim 2600,1760,1720$, $1640,1610,1505 .{ }^{1} \mathrm{H}-\mathrm{NMR}\left(\mathrm{CD}_{3} \mathrm{COCD}_{3}\right) \delta: 2.24(3 \mathrm{H}, \mathrm{s})$, $2.25(3 \mathrm{H}, \mathrm{s}), 2.28(3 \mathrm{H}, \mathrm{s}), 2.29(3 \mathrm{H}, \mathrm{s}), 3.22(1 \mathrm{H}, \mathrm{dd}, J=$ $8.5,14.5 \mathrm{~Hz}), 3.33(1 \mathrm{H}, \mathrm{dd}, J=4.2,14.5 \mathrm{~Hz}), 5.34(1 \mathrm{H}$, $\mathrm{dd}, J=4.2,8.5 \mathrm{~Hz}), 6.59(1 \mathrm{H}, \mathrm{d}, J=16.1 \mathrm{~Hz}), 7.18(1 \mathrm{H}, \mathrm{d}$, $J=8.1 \mathrm{~Hz}), 7.27(1 \mathrm{H}, \mathrm{d}, J=2.0 \mathrm{~Hz}), 7.29(1 \mathrm{H}, \mathrm{dd}, J=2.0$, $8.1 \mathrm{~Hz}), 7.32(1 \mathrm{H}, \mathrm{d}, J=9.0 \mathrm{~Hz}), 7.62(1 \mathrm{H}, \mathrm{d}, J=2.0 \mathrm{~Hz})$, $7.63(1 \mathrm{H}, \mathrm{dd}, J=2.0,9.0 \mathrm{~Hz}), 7.68(1 \mathrm{H}, \mathrm{d}, J=16.1 \mathrm{~Hz})$. ${ }^{13} \mathrm{C}-\mathrm{NMR}\left(\mathrm{CD}_{3} \mathrm{COCD}_{3}\right) \delta: 170.6,168.64(2 \mathrm{C}), 168.59$, $168.48,166.1,145.1,144.5,143.9,143.2,142.3,136.1$, $133.9,128.1127 .4,125.6,124.9,124.2,124.1,119.3,73.3$, $37.2,20.5(4 \mathrm{C})$. MS m/z: $528\left(\mathrm{M}^{+}, \mathrm{C}_{26} \mathrm{H}_{24} \mathrm{O}_{12}\right), 486,468$, $444,426,402,384,282,264,247,240,222,205,198,180$, $163,123$.

Methylation of compound $\mathbf{4 b}$. A solution of compound $\mathbf{4 b}(10 \mathrm{mg})$ in ether $(2 \mathrm{ml})$ was treated with ethereal diazomethane at $4^{\circ} \mathrm{C}$ for $5 \mathrm{~min}$. After the excess diazomethane had been decomposed with a few drops of acetic acid, the reaction mixture was extracted with aq. saturated $\mathrm{NaHCO}_{3}$ solution. The ethereal layer was washed with water, dried over anhydrous $\mathrm{MgSO}_{4}$ and concentrated in vacuo. The crude methylated product $(8 \mathrm{mg})$ was recrystallized from $\mathrm{MeOH}$ to give colorless needles of compound $4 \mathrm{c}, \operatorname{mp} 99 \sim 101^{\circ} \mathrm{C}$. UV $\lambda_{\max }\left(\mathrm{CHCl}_{3}\right) \mathrm{nm}(\log \varepsilon)$ : $284(4.35) . \quad[\alpha]_{\mathrm{D}}^{25}:+35.0^{\circ} \quad\left(c=0.2, \mathrm{CHCl}_{3}\right)$. IR $v_{\max }$ (Nujol) $\mathrm{cm}^{-1}: 1760,1720,1680,1630,1500,1200 .{ }^{1} \mathrm{H}-$ NMR $\left(\mathrm{CDCl}_{3}\right) \delta: 2.270(3 \mathrm{H}, \mathrm{s}), 2.274(3 \mathrm{H}, \mathrm{s}), 2.30(3 \mathrm{H}, \mathrm{s})$, $2.31(3 \mathrm{H}, \mathrm{s}), 3.18(1 \mathrm{H}, \mathrm{dd}, J=5.1,14.8 \mathrm{~Hz}), 3.23(1 \mathrm{H}, \mathrm{dd}$, $J=7.6,14.8 \mathrm{~Hz}), 3.74(3 \mathrm{H}, \mathrm{s}), 5.35(\mathrm{lH}, \mathrm{dd}, J=5.1$, $7.6 \mathrm{~Hz}), 6.41(1 \mathrm{H}, \mathrm{d}, J=15.9 \mathrm{~Hz}), 7.10 \sim 7.15(3 \mathrm{H}, \mathrm{m}), 7.23$ $(\mathrm{lH}, \mathrm{d}, J=8.5 \mathrm{~Hz}) 7.38(1 \mathrm{H}, \mathrm{d}, J=2.0 \mathrm{~Hz}), 7.42(1 \mathrm{H}, \mathrm{dd}$, $J=2.0,8.5 \mathrm{~Hz}), 7.63(1 \mathrm{H}, \mathrm{d}, J=15.9 \mathrm{~Hz}) .{ }^{13} \mathrm{C}-\mathrm{NMR}$ $\left(\mathrm{CDCl}_{3}\right) \delta: 169.8,168.2,168.1,168.0,167.9,165.6,144.3$, $143.8,142.5,142.0,141.2,134.6,133.1,127.3,126.6,124.5$, $123.9,123.4,123.0,118.2,76.7,52.5,36.8,20.63(2 \mathrm{C})$, $20.59(2 \mathrm{C})$. MS m/z (\%): $542\left(\mathrm{M}^{+}, \mathrm{C}_{27} \mathrm{H}_{26} \mathrm{O}_{12}\right), 500,458$ (5), 440, 416, 398, $278(5), 247(4), 236(17), 222(5), 205$ (19), $194(100), 180(23), 163(48), 123(17)$.

Compound 5a. The second fraction $(70 \mathrm{mg})$ was rechromatographed on a Sephadex LH-20 column using $80 \% \mathrm{EtOH}$ as an eluent to afford an amorphous powder, which was homogeneous on a TLC plate with a solution of AcOEt-MeOH- $\mathrm{H}_{2} \mathrm{O}(21: 4: 3 \mathrm{v} / \mathrm{v})$ as the developing solvent, but was revealed to consist of three components by HPLC analysis: column, develosil 5-ODS (Chemco), $150 \times 4.6 \mathrm{~mm}$; solvent, $\mathrm{H}_{2} \mathrm{O}-\mathrm{MeOH}-\mathrm{HOAc}(27: 23: 1 \mathrm{v} / \mathrm{v})$; flow rate, $0.5 \mathrm{ml} / \mathrm{min}$; pressure, $120 \mathrm{~kg} / \mathrm{cm}^{2}$; detection, absorption at $290 \mathrm{~nm}$. These three constituents were separated by repeated preparative $\mathrm{HPLC}$ using the following system: column, develosil 5-ODS (Chemco), $250 \times 10 \mathrm{~mm}$; solvent, $\mathrm{H}_{2} \mathrm{O}-\mathrm{MeOH}-\mathrm{OHAc}(27: 23: 1 \mathrm{v} / \mathrm{v})$; flow rate, $1.5 \mathrm{ml} / \mathrm{min}$; pressure, $130 \mathrm{~kg} / \mathrm{cm}^{2}$. The major component (5a) was isolated as an off-white amorphous powder (21 mg), mp $152 \sim 155^{\circ} \mathrm{C}$. UV $i_{\max }(\mathrm{MeOH}) \mathrm{nm}(\log \varepsilon): 290$ $(4.07), 328(4.08) \cdot[\alpha]_{\mathrm{D}}^{25}:+76.7^{\circ}(c=0.30, \mathrm{MeOH})$. IR $v_{\max }$ (Nujol) $\mathrm{cm}^{-1}: 3500,3100 \sim 2400,1680,1620,1600,1510$. SI-MS: $559(\mathrm{M}+\mathrm{H}+\mathrm{G})^{+}, 467(\mathrm{M}+\mathrm{H})^{+}, 163,107$.

Acetylation of compound 5a. Acetylation of 5a (15 mg) was carried out as already mentioned. The crude product was purified by column chromatography on Sephadex LH-20 using isopropyl alcohol as an eluent to give a colorless viscous gum (5b). UV $i_{\max }(\mathrm{MeOH}) \mathrm{nm}(\log \varepsilon)$ : 276 (4.29). $[\alpha]_{\mathrm{D}}^{25}:+45.5^{\circ}(c=0.11, \mathrm{MeOH})$. IR $v_{\max }$ (Nujol) $\mathrm{cm}^{-1}: 3400 \sim 2600,1760,1720,1700,1680,1635$, $1590,1505,1200 .{ }^{1} \mathrm{H}-\mathrm{NMR}\left(\mathrm{CD}_{3} \mathrm{COCD}_{3}\right) \delta: 2.22(3 \mathrm{H}, \mathrm{s})$, $2.24(6 \mathrm{H}, \mathrm{s}), 2.29(3 \mathrm{H}, \mathrm{s}), 2.30(3 \mathrm{H}, \mathrm{s}), 3.20(1 \mathrm{H}, \mathrm{dd}$, $J=9.5,14.8 \mathrm{~Hz}), 3.37(1 \mathrm{H}, \mathrm{dd}, J=3.9,14.8 \mathrm{~Hz}), 4.19(2 \mathrm{H}$, s), $5.26(1 \mathrm{H}, \mathrm{dd}, J=3.9,9.5 \mathrm{~Hz}), 6.57(1 \mathrm{H}, \mathrm{d}, J=16.1 \mathrm{~Hz})$, $7.00(1 \mathrm{H}, \mathrm{s}), 7.06(2 \mathrm{H}, \mathrm{d}, J=8.5 \mathrm{~Hz}), 7.24(2 \mathrm{H}, \mathrm{d}$, $J=8.5 \mathrm{~Hz}), 7.29(1 \mathrm{H}, \mathrm{s}), 7.33(1 \mathrm{H}, \mathrm{d}, J=8.8 \mathrm{~Hz}), 7.62(1 \mathrm{H}$, $\mathrm{d}, J=2.0 \mathrm{~Hz}), 7.63(1 \mathrm{H}, \mathrm{dd}, J=2.0,8.8 \mathrm{~Hz}), 7.68(1 \mathrm{H}, \mathrm{d}$, $J=16.1 \mathrm{~Hz}) .{ }^{13} \mathrm{C}-\mathrm{NMR}\left(\mathrm{CD}_{3} \mathrm{COCD}_{3}\right) \delta: 170.8,169.6$, $168.7,168.6(2 \mathrm{C}), 168.5,166.1,150.4,145.1,144.5,143.8$, $142.2,141.6,138.8,138.3,134.3,133.9,130.5(2 \mathrm{C}), 127.4$, $126.6,125.8,124.9,124.1,122.7(2 \mathrm{C}), 119.2,73.1,38.0$, 34.3, 20.9, $20.5(4 \mathrm{c})$. SI-MS: $677(\mathrm{M}+\mathrm{H})^{+}, 163,149,123$, 107.

Methylation of compound $\mathbf{5 b}$. Methylation of compound $\mathbf{5 b}(10 \mathrm{mg})$ was carried out as already mentioned to give a colorless viscous gum $(9 \mathrm{mg})$. UV $\hat{\lambda}_{\max }(\mathrm{MeOH}) \mathrm{nm}(\log \varepsilon)$ : 279 (4.36). $[\alpha]_{\mathrm{D}}^{25}:+22.9^{\circ}\left(c=0.70, \mathrm{CHCl}_{3}\right)$. IR $v_{\max }$ (film) $\mathrm{cm}^{-1}: 1760,1730,1710,1680,1630,1500,1200 .{ }^{1} \mathrm{H}-$ NMR $\left(\mathrm{CDCl}_{3}\right) \delta: 2.24(3 \mathrm{H}, \mathrm{s}), 2.25(3 \mathrm{H}, \mathrm{s}), 2.28(3 \mathrm{H}, \mathrm{s})$, $2.30(3 \mathrm{H}, \mathrm{s}), 2.31(3 \mathrm{H}, \mathrm{s}), 3.13(1 \mathrm{H}, \mathrm{dd}, J=9.0,14.7 \mathrm{~Hz})$, 3.21 ( $1 \mathrm{H}$, dd, $J=4.6,14.7 \mathrm{~Hz}), 3.74(3 \mathrm{H}, \mathrm{s}), 4.07(2 \mathrm{H}, \mathrm{s})$, $5.24(1 \mathrm{H}, \mathrm{dd}, J=4.6,9.0 \mathrm{~Hz}), 6.37(1 \mathrm{H}, \mathrm{d}, J=16.1 \mathrm{~Hz})$, $6.90(1 \mathrm{H}, \mathrm{s}), 7.02(2 \mathrm{H}, \mathrm{d}, J=8.5 \mathrm{~Hz}), 7.11(1 \mathrm{H}, \mathrm{s}), 7.14$ $(2 \mathrm{H}, \mathrm{d}, J=8.5 \mathrm{~Hz}), 7.23(1 \mathrm{H}, \mathrm{d}, J=8.3 \mathrm{~Hz}), 7.36(1 \mathrm{H}, \mathrm{d}$, $J=2.0 \mathrm{~Hz}), 7.40(1 \mathrm{H}, \mathrm{dd}, J=2.0,8.3 \mathrm{~Hz}), 7.62(1 \mathrm{H}, \mathrm{d}$, $J=16.1 \mathrm{~Hz}) .{ }^{13} \mathrm{C}-\mathrm{NMR}\left(\mathrm{CDCl}_{3}\right) \delta: 170.0,169.5,168.2$, $168.1,168.0,167.9,165.7,149.3,144.3,143.8,142.5,141.0$, $140.5,137.7,136.8,133.1,132.9,129.9(2 \mathrm{C}), 126.6,125.3$, $125.2,123.9,123.0,121.8(2 \mathrm{C}), 118.1,72.4,52.5,37.7,33.9$, $21.1,20.7(2 \mathrm{C}), 20.6(2 \mathrm{C})$.

Antioxidative assay. Antioxidative activity was measured by the ferric thiocyanate method as previously described. ${ }^{5)}$

Acknowledgments. This study was supported in part by a Grant-in-Aid for Special Research Project (No. 60216021) from the Ministry of Education, Science and Culture of Japan. We thank Dr. K. Hayashi and Dr. K. Kogami of Hasegawa Perfumery Co., Ltd. for supplying oregano. 


\section{References}

1) J. R. Chipault, G. R. Mizuno, J. M. Hawakins and W. O. Lundberg, Food Res., 17, 46 (1952).

2) J. R. Chipault, G. R. Mizuno and W. O. Lundberg, Food Technol, 10, 209 (1956).

3) J. R. Chipault, Food Engineering, April 1957, 134.

4) N. Nakatani and H. Kikuzaki, Agric. Biol. Chem., 51, 2727 (1987).

5) R. Inatani, N. Nakatani and H. Fuwa, Agric. Biol. Chem., 47, 521 (1983).

6) C. J. Kelley, J. R. Mahajan, L. C. Brooks, L. A.
Neubert, W. R. Breneman and M: Carmack, J. Org. Chem., 40, 1804 (1975).

7) C. J. Kelley, R. C. Harruff and M. Carmack, J. Org. Chem., 41, 449 (1976).

8) M. Aritomi, J. Home Econ. Jpn., 33, 353 (1982).

9) W. R. Woolfenden and D. M. Grant, J. Am. Chem. Soc., 88, 1496 (1966).

10) P. C. Lauterbur, J. Am. Chem. Soc., 83, 1846 (1961).

11) R. Waack, M. A. Doran, E. B. Baker and G. A. Olah, J. Am. Chem. Soc., 88, 1272 (1966).

12) M. L. Scarpati and G. Oriente, Ric. Sci., 30, 255 (1960). 